\title{
ATRAS: adaptive MAC protocol for efficient and fair coexistence between radio over fiber-based and CSMA/CA-based WLANs
}

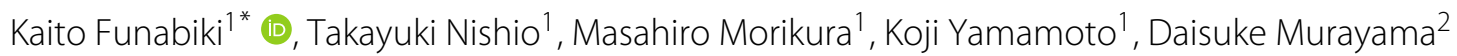 \\ and Katsuya Nakahira ${ }^{2}$
}

\begin{abstract}
In this paper, we propose a medium access control (MAC) protocol to allow a radio over fiber-based wireless local area network (RoF-based WLAN) to coexist with legacy carrier sense multiple access with collision avoidance (CSMA/CA)-based WLANs. In RoF-based WLANs, there are long propagation delays between access points (APs) and stations (STAs). When an RoF-based WLAN uses the conventional CSMA/CA protocol and coexists with legacy WLANs, the propagation delay causes unexpected frame collisions and unfairness between the RoF-based WLAN and legacy WLANs. The proposed protocol reduces unexpected frame collisions and improves fairness between the RoF-based WLAN and legacy WLANs in environments where they coexist. It is only necessary to apply the proposed protocol to the AP in the RoF-based WLAN (RoF AP); there is no need to modify STAs, which is an advantage of the protocol. In the proposed scheme, the RoF AP transmits frames during the transmission and reception of frames in the legacy WLAN, such that the frame transmitted by the AP arrives at a destination STA one short interframe space (SIFS) period after the channel became idle, and thus the RoF AP's frames do not collide with other frames. As a result, the proposed method decreases the likelihood of frame collisions and increases the throughput of the RoF-based WLAN. In addition, the proposed method provides adaptive adjustment of the transmission probability, which enables the RoF-based WLAN and the legacy WLANs to fairly share wireless channels. The proposed protocol has been investigated for IEEE $802.11 \mathrm{a} / \mathrm{b} / \mathrm{g}$ WLANs. Numerical analysis and simulation evaluations show that the proposed scheme increases the TCP throughput of the RoF-based WLAN up to the same level as that in legacy WLANs.
\end{abstract}

Keywords: Radio over fiber, Long distance WLAN, Coexistence, MAC protocol, Propagation delay

\section{Introduction}

Wireless communication is becoming increasingly common, and many wireless standards are now available for wireless local area networks (WLANs). Accordingly, network operators must prepare dedicated wireless access points (APs) for each system, every time a new wireless standard emerges. Existing WLAN standards, such as IEEE $802.11 \mathrm{~b} / \mathrm{g} / \mathrm{a} / \mathrm{n} / \mathrm{ac}$, are utilized in the 2.4 and $5 \mathrm{GHz}$ bands. Moreover, owing to increasing demand for more capacity, WLAN standards using the millimeterwave (mmWave) band, such as IEEE 802.11ad and IEEE 802.11ay using $60 \mathrm{GHz}$, are actively being discussed for

*Correspondence: nishio@i.kyoto-u.ac.jp

${ }^{1}$ Graduate School of Informatics, Kyoto University, Kyoto, Japan

Full list of author information is available at the end of the article the provision of broadband wireless access [1]. In addition, the demand for Internet of Things (IoT) applications has drawn attention to sub- $1 \mathrm{GHz}$ wireless standards, such as IEEE 802.15.4 [2]. The upcoming IEEE 802.11ah standard has the potential to serve wide-area and low-cost wireless networks for IoT devices [3].

Access networks are required to support various communication standards with different physical (PHY) and medium access control (MAC) layer parameters by a single AP. Network function virtualization (NFV) is a promising method with which to economically realize such flexible wireless access networks [4].

One of the key enablers of NFV for wireless access networks is radio over fiber (RoF) technology. In wireless systems with RoF technology, only radio frequency (RF) units connected to a central office are located in remote 
areas and the central office operates the PHY signal processing and MAC functionality [5-9]. The RF units, which are connected to the central office via an optical fiber, convert the optical signals to electrical signals before transmitting them over the radio channel, and vice versa. Using RoF for wireless systems enables the centralization of the wireless signal processing functions that are currently performed by each AP. The benefit of this approach is that WLANs or wireless personal area networks (WPANs) are able to support any wireless standard by only changing the processing functions at the central office.

An RoF-based access architecture for integrating multiple wireless systems was discussed in [8, 9]. However, the integration of the conventional carrier sense multiple access with collision avoidance (CSMA/CA)-based wireless standard with an RoF-based architecture requires research because the long propagation delays in the optical fiber links may cause the CSMA/CA protocol to behave unexpectedly, resulting in a decrease in the system throughput. When designing a MAC protocol for RoFbased WLANs, it is important to take into account backward compatibility with IEEE 802.11 standard to cover existing devices. Moreover, it is also important to take into account how best to support coexistence with legacy CSMA/CA-based systems. WLANs are used widely and many APs are densely deployed. The dense environment is typical for apartment buildings and offices. Because of the limited number of orthogonal frequency channels in 2.4 and $5 \mathrm{GHz}$ band, situations where the neighboring WLANs utilizing the same channel can occur. This paper focuses on a MAC layer solution for IEEE 802.11 WLANs that employ an RoF architecture (RoF-based WLAN) and addresses the coexistence problems between the RoF-based and legacy WLANs.

In WLANs that have an RoF-based architecture, delays longer than one half of a slot time cause unexpected frame collision patterns and increase the probability of frame collisions when the WLAN operates using a conventional MAC protocol. In order to solve the severe frame collision problems caused by large propagation delays, several alternative MAC protocols have been proposed [10-12]. For example, Deronne et al. proposed a method for optimizing the slot time value so that unexpected collisions do not occur [10]. However, the use of large slot times increases the overhead, which decreases the system throughput. Moreover, if an RoF-based WLAN coexists with legacy WLANs, the RoF-based WLAN stations that use a large slot time are only able to transmit a few DATA frames because their slot time is much larger than that of the legacy WLAN stations. Other schemes described in $[11,12]$ improve the throughput of RoF-based WLANs by using the frame aggregation and BlockACK mechanisms. However, these schemes do not solve the frame collision problems caused by long propagation delay because frame aggregation does not reduce the number of frame collisions, not to mention that there are still open issues concerning the coexistence of RoF and legacy WLANs.

In this paper, we propose a new MAC protocol for an AP, called "advanced transmission for RoF-based WLAN system" scheme. The ATRAS scheme has been investigated for IEEE 802.11a/b/g WLANs. The ATRAS scheme solves the unfairness and TCP throughput degradation problems by increasing the number of transmission opportunities for an AP in RoF-based WLANs (RoF AP). Furthermore, the ATRAS scheme is able to control the throughput performance of an RoF-based WLAN by changing the transmission probability of the RoF AP. We also provide a mathematical throughput model of the coexistence environment, as well as an adaptive transmission probability control scheme for the RoF AP using the ATRAS scheme.

The contributions of this paper are as follows: (1) a design for an adaptive MAC protocol for RoF APs that enables fair and efficient coexistence without modification of the STAs and legacy WLANs; as far as we know, there is no MAC protocol suitable for the coexistence environment, (2) a mathematical throughput model of the proposed scheme and parameters determined based on the throughput model, and (3) numerical evaluations using the network simulator QualNet [13].

This paper is organized as follows. Section 2 introduces a system model of the coexistence environment and provides a problem statement. Section 3 describes related works. In Section 4, we describe how the proposed MAC protocol improves the throughput of an RoF-based WLAN, and then introduce a mathematical throughput model of the same. Section 5 shows the results of numerical evaluations performed by computer simulations, and our conclusions provided in Section 6.

\section{RoF-based WLAN}

\subsection{System model}

In this paper, we consider an environment where an RoFbased WLAN coexists with legacy WLANs, as shown in Fig. 1. The IEEE 802.11 standard is used for the WLANs and the WLANs are operated in the same frequency channel in the 2.4 or $5 \mathrm{GHz}$ band.

The WLAN STAs associated with the legacy AP are denoted as legacy STAs, while the WLAN STAs associated with the RoF AP are denoted as RoF STAs. Furthermore, we define "local nodes" as the legacy APs, legacy STAs, and RoF STAs. All STAs operate according to the IEEE 802.11 standard MAC protocol [14].

The RoF AP in the RoF-based WLAN consists of an RF module in a premises of the customer and a processing unit located at a central office. The RF module is composed of an antenna, an amplifier, an analog-to-digital $(\mathrm{AD}) /$ digital-to-analog (DA) converter, and an interface 


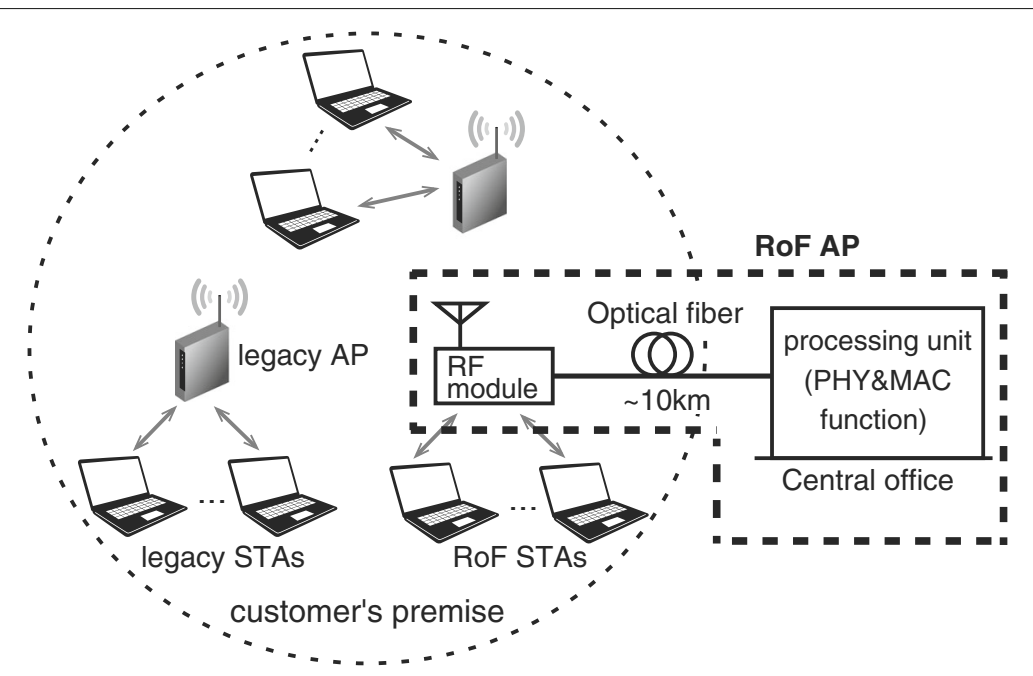

Fig. 1 Coexistence of RoF-based WLAN and legacy WLANs. Legacy APs and STAs, RoF STAs, and RF unit of RoF AP are deployed densely

circuit, and is connected to a centralized processing unit via an optical fiber. The processing unit handles the PHY and MAC functions for an AP. In this system, the frames transmitted by an STA are received by the RF module, and then amplified, down-converted, digitized, and converted into optical signals before being transmitted through the optical fiber to the processing unit located at the central office. We assume that distance between the RF module of the RoF AP and the other nodes is several meters to several hundred meters and the propagation delay of wireless signal is negligible.

It should be noted that there is a non-negligible propagation delay $\delta_{\text {RoF }}$ between the processing unit and STAs due to the presence of the optical links. In Japan, most telephone subscribers are located within $5 \mathrm{~km}$ of a telecommunication office. In order to accommodate most customers, our simulation assumed a maximum fiber length of $10 \mathrm{~km}$, which corresponds to a one-way propagation delay of $50 \mu \mathrm{s}$. This delay can be derived from the refractive index of single-mode optical fiber, i.e., 1.5 [15].

\subsection{Delay-induced issues on CSMA/CA}

In this section, we discuss some of the issues that impair the throughput of RoF-based WLANs and degrade the fairness between an RoF-based WLAN and legacy WLANs in environments where they coexist.

\subsubsection{Increase in collision between frames transmitted by RoF AP and local nodes}

In the RoF-based WLAN, frames transmitted by the RoF AP or STAs are received after $\delta_{\text {RoF }}$. The long propagation delay causes a large displacement in the carriersense timing at the receive side. This phenomenon causes the carrier-sense mechanism to underperform, causes unexpected collisions, and increases the probability of collisions.

In the RoF-based WLAN, frames transmitted in different slot times could collide while collisions between frames only occur if multiple nodes transmit in the same slot time in an ordinary WLAN with a small propagation delay. Figure 2 depicts a frame collision event between an RoF AP and local nodes. In the figure, a local node transmits an ACK frame, and after the ACK frame transmission, an RoF AP transmits a DATA frame based on a conventional distributed coordination function (DCF) in the $k$ th slot time. The frames transmitted by the RoF AP in the $k$ th slot time could collide with a frame transmitted by a local node in the $k$ th and other slot times, the $(k+1)$ th or $(k+2)$ th slot time in the figure. In the $(k+3)$ th slot time, local nodes can detect signal transmitted by the RoF AP, thus local nodes suspend their transmission. This phenomenon increases the frame collision probability.

When TCP downlink traffic is the dominant type of traffic in the RoF-based and legacy WLANs, TCP-DATA frames transmitted by the RoF AP could suffer from the frame collisions. Accordingly, this severely degrades the TCP throughput performance of the RoF-based WLAN.

\subsubsection{ACK frame collision}

In a conventional DCF, acknowledge (ACK) frames never collide with other frames because the ACK frames are transmitted after the short interframe space (SIFS) period. However, the ACK frames may collide with other frames in the RoF-based WLAN owing to the long propagation delays.

Figure 3 illustrates a collision between an ACK frame from an RoF AP and a frame transmitted by a local node, where the RoF AP replies ACK frame to an RoF STA and 


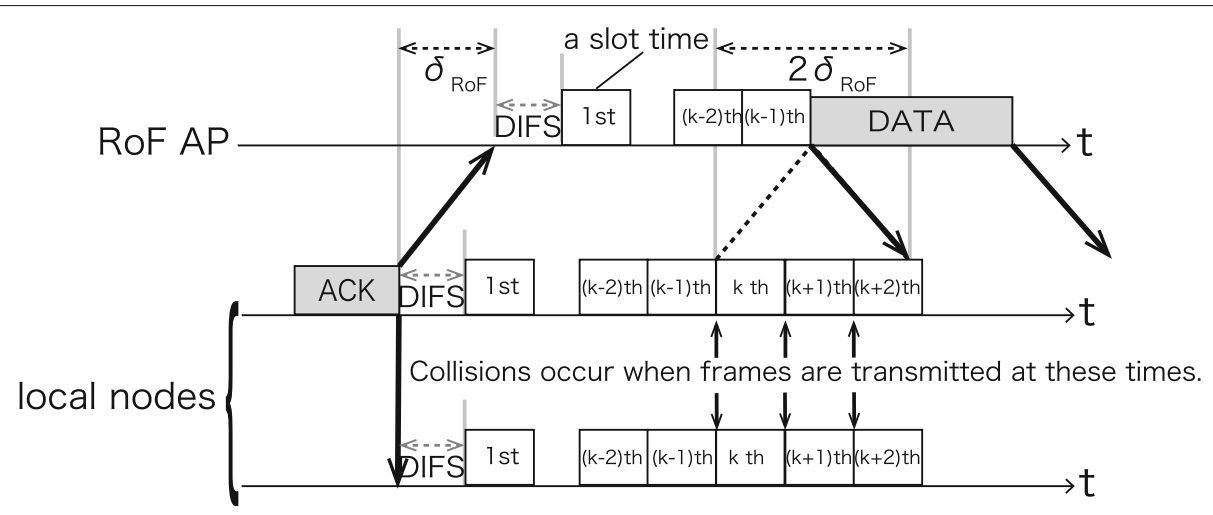

Fig. 2 Frame collision between RoF AP and local nodes

a local node transmits a DATA frame before the ACK is received by the RoF STA, which collides with the ACK frame. Although local nodes wait for a network allocation vector (NAV) period indicated in the DATA frame transmitted by the RoF STA before transmitting their frames, long propagation delays cause the NAV period to expire and the local noses could transmit frames before the RoF STA receives the ACK frame from the RoF AP. The ACK collision increases the number of retransmission in the RoF-based WLAN and degrades the throughput performance of the RoF-based WLAN.

\section{Related works}

\subsection{Slot time adjustment scheme}

One method to address this problem is to optimize the slot time value to account for the propagation delay. Deronne et al. proposed a method for optimizing the slot time value of RoF-based WLANs [10]. The original slot time value $\sigma$ is defined in the IEEE 802.11 standard [14] as aCCATime + aRxTxTurnaroundTime + aAirPropagationTime + aMACProcessingDelay, where aCCATime is the time required to determine the state of the channel, aRxTxTurnaroundTime is the time required to change from receive to transmit, aAirPropagationTime is the roundtrip air propagation time, and aMACProcessingDelay is the time needed for

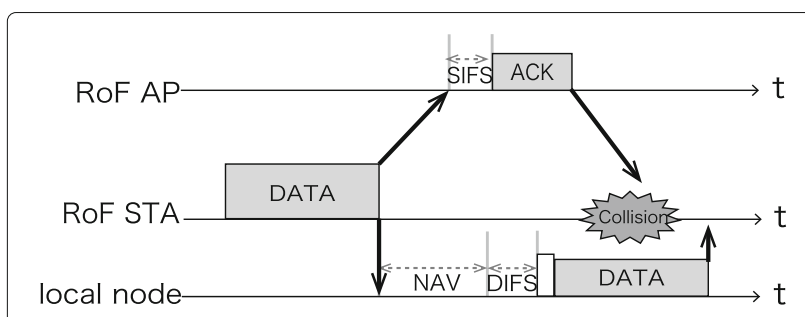

Fig. 3 ACK frame collision. An ACK frame transmitted by an RoF AP collides with a DATA frame transmitted by a local node computations in the MAC layer. In [10], the slot time value was adapted according to the following formula: Slottime $=\sigma+2\left(\delta_{\mathrm{RoF}}-4.5 \mu \mathrm{s}\right)$. Adapting the slot time value decreases the number of unexpected frame collisions mentioned in Section 2.2.1 since the carrier-sense mechanism still functions normally.

However, extending the slot time increases the overhead duration in CSMA/CA, which leads to unfairness between an RoF-based WLAN and a legacy WLAN. For example, when the propagation delay is $50 \mu \mathrm{s}$, the slot time value must be greater than $100 \mu \mathrm{s}$, which is 11 times longer than the original slot time of $9 \mu \mathrm{s}$ in the IEEE 802.11a standard [14]. Moreover, when an RoF-based WLAN uses an extended slot time value but a coexisting legacy WLAN employs the usual slot time, the nodes in the RoF-based WLAN are not able to transmit enough frames because their backoff period is much longer than that in the legacy WLAN, as shown in Fig. 4. This results in serious throughput degradation in the RoF-based WLAN.

\subsection{Piggy back access scheme}

In our previous work [16], we presented a transmission scheme to increase the number of successful RoF AP frame transmissions by applying a piggy back access (PBA) scheme [17] to an RoF AP in an environment where only

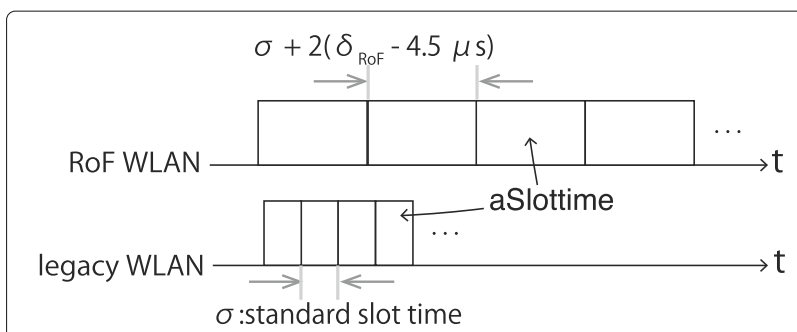

Fig. 4 Backoff time in slot time adjustment scheme. Backoff time of the RoF-based WLAN becomes much larger than that of the legacy WLAN 
RoF-based WLANs exist. In that case, the RoF AP transmits a DATA frame after a SIFS period instead of after a DIFS period plus the backoff time when the RoF AP is transmitting an ACK frame to an STA. The RoF STA receives the DATA frame one SIFS period after the RoF STA received the ACK frame so that the RoF AP's DATA frame does not collide.

This PBA scheme works well in a bidirectional traffic scenario where RoF STAs frequently transmit frames; however, since TCP downlink traffic is dominant in actual wireless Internet access [18], where STAs do not have full buffer traffic, the throughput performance of the PBA scheme may decrease in a real-world wireless Internet access environment.

\subsection{NAV extension method}

To solve the ACK frame collision problem in Section 2.2.2, we also proposed extending the NAV period by using the request-to-send (RTS)/ clear-to-send (CTS) procedure for an RoF-based WLAN [16], as illustrated in Fig. 5. When an RoF AP receives an RTS frame, the RoF AP replies with a CTS frame that includes the extended NAV duration. Specifically, the NAV duration value of the CTS frame transmitted by the RoF AP $T_{\text {exNAV }}$ is defined as $T_{\text {exNAV }}=$ $T_{\mathrm{NAV}, \mathrm{st}}+3 \delta_{\mathrm{RoF}}$, where $T_{\mathrm{NAV}, \mathrm{st}}$ is the conventional NAV duration of a CTS frame defined in the IEEE 802.11a standard [14]. This method prevents ACK frame collisions and is backwards compatible.

\subsection{Collision avoidance using TDMA fashion}

Collision free MAC protocols exploiting a TDMA fashion have been studied to solve collision problems in WLANs [19-25]. In these protocol, there are periods to transmit frames in a TDMA fashion, where APs and STAs can transmit frames without collision. Allocating TDMA timeslots to RoF APs taking into account the large delay could avoid delay induced collisions. However, these approaches require major modifications to a MAC protocol of the STAs, which means that conventional devices can not be used in the WLANs using the protocol exploiting a TDMA fashion. Our protocol focuses on RoF-based WLAN with backward compatibility with IEEE 802.11 standard, where existing WLAN devices are available. Therefore, these approaches exploiting TDMA are not suitable for our target application.

\section{Proposed scheme (ATRAS)}

In this section, we describe the proposed ATRAS method and its application only to the RoF AP. In this method, we assume that the RoF STAs use the conventional DCF procedure except with respect to the values of an ACK timeout interval and a CTS timeout interval. To prevent frame transmission failure due to timeout, it is necessary to extend the ACK and CTS timeout intervals of the RoF STAs. The timeout interval $T_{\text {timeout }}$ should be set to the value obtained from $T_{\text {timeout }}=T_{\mathrm{st}}+2 \delta_{\mathrm{RoF}}$, where $T_{\mathrm{st}}$ is a conventional timeout interval used in IEEE 802.11. The values of the ACK and CTS timeout intervals can be changed by setting or updating driver software [26, 27].

In this section, we describe the transmission procedures of the RoF AP in the ATRAS scheme and the theoretical model of the total throughput for UDP bidirectional full buffer traffic. We also explain the adaptive control scheme employing the transmission probability $\alpha(0<\alpha \leq 1)$, the initial value of which is derived from the theoretical throughput model.

\subsection{Basic procedure of the ATRAS scheme}

In order to solve the RoF AP frame collision problem shown in Fig. 2 in the environment where an RoFbased WLAN and legacy WLANs coexist, we propose the ATRAS scheme. The procedures in the ATRAS scheme are performed when the RoF AP overhears a CTS frame transmitted by a node in a legacy WLAN, or when the RoF AP overhears a DATA frame transmitted by a node in a legacy WLAN not using RTS/CTS. Figure 6 depicts the ATRAS scheme when a legacy WLAN uses RTS/CTS. The following sections explain and evaluate the proposed scheme for a case where legacy WLANs use RTS/CTS. The RoF AP receiving a CTS frame transmitted by a legacy

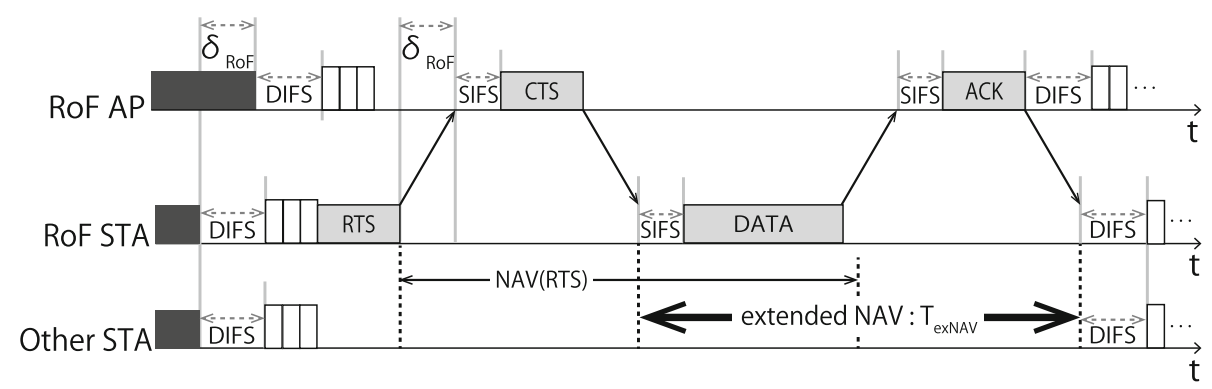

Fig. 5 NAV extension method in an RoF-based WLAN. A CTS frame transmitted by the RoF AP extends NAV at STAs 


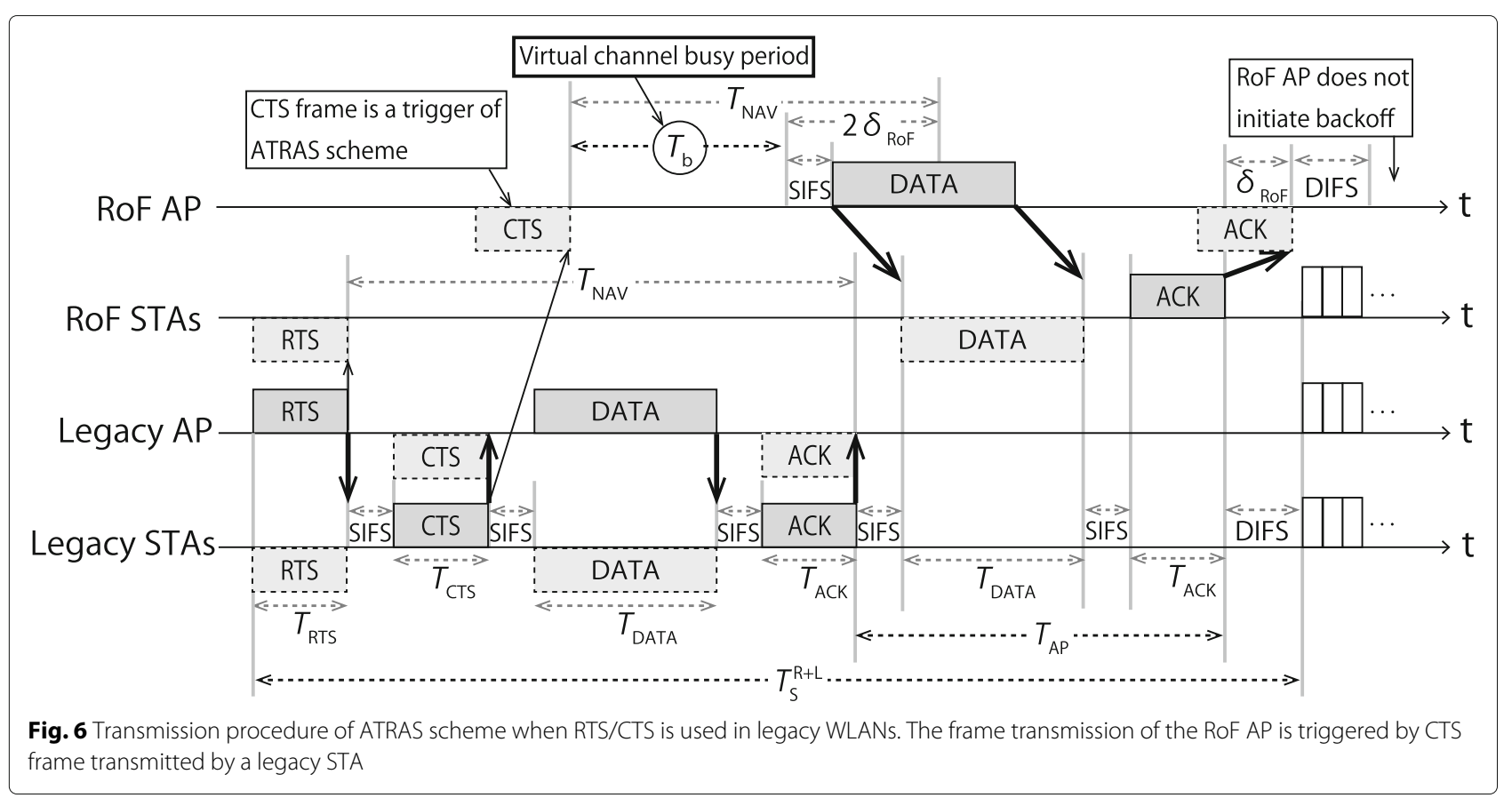

STA sets a virtual channel busy period, the duration $T_{\mathrm{b}}$ of which is defined as $T_{\mathrm{b}}=T_{\mathrm{NAV}}-2 \delta_{\mathrm{RoF}}$, where $T_{\mathrm{NAV}}$ is the duration of the NAV period. The virtual channel busy period is introduced to avoid collision between frames transmitted by a local node and the RoF AP. If the RoF AP transmits a frame in the virtual channel busy period, the frame collides with a frame transmitted by a local node. The RoF AP transmits its DATA frame SIFS period after the virtual channel busy period with the probability $\alpha$, which is introduced to control throughput of the RoF AP. DATA frames transmitted by the RoF AP using the ATRAS scheme are received by local nodes SIFS period after the channel actually becomes idle. Therefore, the frame collision mentioned in Section 2.2.1 does not occur.

The parameter $\alpha$ represents the frame transmission probability, and the WLAN operator at the central office can control the downlink throughput of the RoF-based WLAN by adjusting this probability so that the channel is fairly shared with the RoF-based WLAN and the legacy WLAN. Introducing transmission probability to control throughput is commonly used approach in WLANs [28]. With the probability $\alpha$, the RoF AP transmits its DATA frame SIFS period after the virtual channel busy period when the RoF AP receives a CTS frame from a legacy AP or STA. With the probability $(1-\alpha)$, the RoF AP does not set a virtual channel busy period but waits for its NAV period $T_{\mathrm{NAV}}$. We will describe the method to adjust the probability in Section 4.3.

The number of transmission opportunities for the RoF AP using the ATRAS scheme depends on $\alpha$ and the traffic volume of the legacy WLANs. When the traffic of the legacy WLANs is low, the RoF AP is not able to obtain many transmission opportunities by the ATRAS scheme. Using backoff or/and piggyback access scheme with the ATRAS scheme could increase transmission opportunity of the RoF AP. For example, it is conceivable that the RoF AP could measure the traffic volume of the legacy WLANs by overhearing frames transmitted by them and determine a certain threshold value, and when the traffic volume exceeded the threshold value, the RoF AP would use the ATRAS scheme. However, such a protocol design of adaptive usage of multiple medium access schemes is out of focus of this paper and we would like to include it to our future work.

\subsection{Theoretical throughput model}

We now develop a total throughput model for legacy WLANs and an RoF-based WLAN with the proposed scheme when they coexist. For simplicity, we provide a theoretical model for UDP bidirectional full buffer traffic, and we determine the initial value of $\alpha$ in the presence of TCP traffic by using an approximated model based on UDP bidirectional full buffer traffic, the details of which are described in the next section. Our model is an extension of a well-known analytical model for conventional WLANs [29].

The theoretical system throughput was derived for a conventional WLAN in [29]:

$$
S=\frac{P_{\mathrm{s}} P_{\operatorname{tr}} \mathrm{E}[P]}{\left(1-P_{\mathrm{tr}}\right) \sigma+P_{\operatorname{tr}} P_{\mathrm{s}} T_{\mathrm{s}}+P_{\operatorname{tr}}\left(1-P_{\mathrm{s}}\right) T_{\mathrm{c}}},
$$


where $P_{\mathrm{s}}$ and $P_{\mathrm{tr}}$ are the probability of successful transmission, and the probability of transmission, respectively, $\mathrm{E}[P]$ is the average packet payload size of the DATA frames, $\sigma$ is the slot time size, and $T_{\mathrm{s}}$ and $T_{\mathrm{c}}$ are the average periods during which the channel is busy due to the successful transmission and collision of frames, respectively.

$$
\begin{aligned}
T_{\mathrm{s}}= & T_{\mathrm{RTS}}+\mathrm{SIFS}+\delta+T_{\mathrm{CTS}}+\mathrm{SIFS}+\delta \\
& +T_{\mathrm{DATA}}+\mathrm{SIFS}+\delta+T_{\mathrm{ACK}}+\delta+\mathrm{DIFS}, \\
T_{\mathrm{c}}= & T_{\mathrm{RTS}}+\mathrm{EIFS}
\end{aligned}
$$

where $T_{\mathrm{RTS}}, T_{\mathrm{CTS}}, T_{\mathrm{DATA}}$, and $T_{\mathrm{ACK}}$ are the required periods for transmitting an RTS frame, a CTS frame, a DATA frame, and an ACK frame, respectively, SIFS, DIFS, and EIFS are the SIFS period, the DIFS period, and the extended inter frame space (EIFS) period defined in [14], respectively, and $\delta$ is the one-way propagation delay in the WLAN.

Based on (1)-(3), we formulate the total system throughput $S^{\mathrm{R}+\mathrm{L}}$ in an environment in which an RoFbased WLAN using the ATRAS scheme coexists with a legacy WLAN, as follows:

$$
S^{\mathrm{R}+\mathrm{L}}=\frac{P_{\mathrm{s}} P_{\mathrm{tr}}\left(1+\alpha^{\prime}\right) \mathrm{E}[P]}{\left(1-P_{\mathrm{tr}}\right) \sigma+P_{\mathrm{tr}} P_{\mathrm{s}} T_{\mathrm{s}}^{\mathrm{R}+\mathrm{L}}+P_{\mathrm{tr}}\left(1-P_{\mathrm{s}}\right) T_{\mathrm{c}}^{\mathrm{R}+\mathrm{L}}}
$$

$$
\begin{aligned}
T_{\mathrm{s}}^{\mathrm{R}+\mathrm{L}}= & T_{\mathrm{RTS}}+\mathrm{SIFS}+T_{\text {delay }}+T_{\mathrm{CTS}}+\mathrm{SIFS} \\
& +T_{\text {delay }}+T_{\mathrm{DATA}}+\mathrm{SIFS}+T_{\text {delay }} \\
& +T_{\mathrm{ACK}}+T_{\text {delay }}+\mathrm{DIFS}+T_{\mathrm{AP}}, \\
T_{\mathrm{AP}}= & \alpha^{\prime}\left(\text { SIFS }+T_{\mathrm{DATA}}+\mathrm{SIFS}+T_{\mathrm{ACK}}\right), \\
T_{\mathrm{c}}^{\mathrm{R}+\mathrm{L}}= & T_{\mathrm{c}}=T_{\mathrm{RTS}}+\text { EIFS } .
\end{aligned}
$$

In contrast to the conventional model, the term $\alpha^{\prime}$ is added into (4), where $\alpha^{\prime}$ is the ratio between the number of additional frame transmissions that use the ATRAS scheme and the number of frame transmissions that use the backoff procedure, as follows:

$$
\alpha^{\prime}=\frac{N_{\mathrm{A}}^{\mathrm{L}}+N_{\mathrm{S}}^{\mathrm{L}}}{N_{\mathrm{A}}^{\mathrm{L}}+N_{\mathrm{S}}^{\mathrm{L}}+N_{\mathrm{S}}^{\mathrm{R}}} \alpha,
$$

where $N_{\mathrm{S}}^{\mathrm{R}}, N_{\mathrm{A}}^{\mathrm{L}}$, and $N_{\mathrm{S}}^{\mathrm{L}}$ are the numbers of RoF STAs, legacy APs, and legacy STAs, respectively. The RoF AP transmits a DATA frame only when it overhears that a
CTS frame has been transmitted by a legacy AP or STA. Thus, the larger ratio of the legacy AP and STAs to the RoF STAs increases the throughput of the RoF AP. When the number of legacy nodes is small, $\alpha$ should be large to obtain large throughput in the RoF AP.

Moreover, in (5), $T_{\mathrm{AP}}$ is the time required for the transmission from the RoF AP, as shown in Fig. 6, and $T_{\text {delay }}$ is the average propagation delay for frames transmitted in the RoF and legacy WLANs. $T_{\text {delay }}$ is calculated using the following equation:

$$
T_{\text {delay }}=\frac{\left(N^{\mathrm{R}}-1\right)}{N} \delta_{\text {RoF }}+\frac{N^{\mathrm{L}}}{N} \delta,
$$

where $N$ is the total number of local nodes, $N^{\mathrm{R}}$ is the total number of RoF APs and RoF STAs, and $N^{\mathrm{L}}$ is the total number of legacy APs and STAs, i.e., $N=N_{\mathrm{A}}^{\mathrm{L}}+N_{\mathrm{S}}^{\mathrm{L}}+N_{\mathrm{S}}^{\mathrm{R}}$, $N^{\mathrm{R}}=N_{\mathrm{A}}^{\mathrm{R}}+N_{\mathrm{S}}^{\mathrm{R}}$, where $N_{\mathrm{A}}^{\mathrm{R}}$ is the number of RoF APs, and $N^{\mathrm{L}}=N_{\mathrm{A}}^{\mathrm{L}}+N_{\mathrm{S}}^{\mathrm{L}}$.

In the ATRAS scheme, the RoF AP does not transmit DATA or RTS frames using the backoff procedure but only transmits DATA frames using the ATRAS scheme. In addition, the frames transmitted by the RoF AP do not collide with other frames from local nodes. Therefore, the AP's transmissions do not affect the procedures in the local nodes in the DCF, and $P_{\mathrm{s}}$ and $P_{\text {tr }}$ can be obtained by using the method that was described in [29]. The total system throughput can be calculated using (4)-(9).

\subsection{Adaptive transmission probability control}

The throughput of an RoF AP can be controlled by adjusting the transmission probability $\alpha$. We control $\alpha$ such that the throughput of the RoF-based WLAN and legacy WLAN converge to the same value in full buffer traffic conditions. In this section, we describe the decision procedure to determine $\alpha$. In this procedure, the initial value of $\alpha$ is determined based on the analytical throughput model; however, the initial value deviates from the optimal value because of traffic fluctuations. Thus, the proposed procedure adaptively adjusts the value of $\alpha$ after the initial setup.

\subsubsection{Objective of the adaptive control}

The objective of the adaptive transmission probability control is that the throughput of an RoF-based WLAN and a legacy WLAN converge to the same amount. We therefore consider the following minimization problem:

$$
\begin{array}{ll}
\underset{\alpha}{\operatorname{minimize}} & \left|S^{R}-S^{L}\right| \\
\text { subject to } & 0 \leq \alpha \leq 1 .
\end{array}
$$

$S^{\mathrm{R}}$ and $S^{\mathrm{L}}$ are the throughput of the RoF-based WLAN and legacy WLAN, respectively. From (4), the throughput 
of an RoF AP $S_{A}^{R}$ can be derived from the following equation:

$$
S_{\mathrm{A}}^{\mathrm{R}}=\frac{P_{\mathrm{s}} P_{\mathrm{tr}} \alpha^{\prime} \mathrm{E}[P]}{\left(1-P_{\mathrm{tr}}\right) \sigma+P_{\mathrm{tr}} P_{\mathrm{s}} T_{\mathrm{s}}^{\mathrm{R}+\mathrm{L}}+P_{\operatorname{tr}}\left(1-P_{\mathrm{s}}\right) T_{\mathrm{c}}^{\mathrm{R}+\mathrm{L}}} .
$$

The total throughput of the local nodes can be calculated by subtracting $S_{\mathrm{A}}^{\mathrm{R}}$ from the total system throughput $S^{\mathrm{R}+\mathrm{L}}$. When the local nodes have the same number of transmission opportunities, the average throughput $s_{i}$ of local node $i$ is shown in (12).

$$
s_{i}=\left(S^{\mathrm{R}+\mathrm{L}}-S_{\mathrm{A}}^{\mathrm{R}}\right) / N \text {. }
$$

Therefore, the values of $S^{\mathrm{R}}$ and $S^{\mathrm{L}}$ can be derived from:

$$
\begin{aligned}
& S^{\mathrm{R}}=S_{\mathrm{A}}^{\mathrm{R}}+\sum_{i \in N_{\mathrm{S}}^{\mathrm{R}}} s_{i}, \\
& S^{\mathrm{L}}=\sum_{i \in N^{\mathrm{L}}} s_{i},
\end{aligned}
$$

where $S^{\mathrm{R}}$ is a summation of the throughput of RoF AP derived from (11) and the throughput of RoF STAs, each of which is derived from (12), and $S^{\mathrm{L}}$ is a summation of throughput of legacy AP and STAs, each of which is also derived from (12).

\subsubsection{Initial value of $\alpha$}

Here, we attempt to find an initial value of $\alpha: \alpha_{0}$ based on (10) for the case of UDP and TCP traffic.

$\alpha_{0}$ under UDP traffic: To accomplish our objective of adaptive control based on (10), we need to determine $\alpha_{0}$ by solving the following equation:

$$
\left|S^{\mathrm{R}}-S^{\mathrm{L}}\right|=0 .
$$

If the above equation is satisfied, then the throughput of the RoF and legacy WLANs are identical. By solving (15) using (8) and (11)-(14), we obtain

$$
\alpha_{0}=\frac{N_{\mathrm{A}}^{\mathrm{L}}+N_{\mathrm{S}}^{\mathrm{L}}-N_{\mathrm{S}}^{\mathrm{R}}}{N_{\mathrm{A}}^{\mathrm{L}}+N_{\mathrm{S}}^{\mathrm{L}}} .
$$

Accordingly, $\alpha_{0}$ is determined by the number of local nodes sending UDP traffic. Since $1 \geq \alpha \geq 0$, when $N_{\mathrm{A}}^{\mathrm{L}}+N_{\mathrm{S}}^{\mathrm{L}} \leq N_{\mathrm{S}}^{\mathrm{R}}$, (15) cannot be satisfied. In the case, the number of RoF STAs should be limited or other fairness criteria should be selected, which is beyond the scope of this paper.

$\alpha_{0}$ under TCP traffic: Bruno et al. reported that TCP traffic results in very low contention in a WLAN because of the TCP flow control algorithm [30]. In the presence of persistent TCP upload and download connections, the average number of STAs that compete for transmission has an upper bound of less than two on average, regardless of the total number of STAs in a WLAN. Based on this result, we can assume that the number of STAs competing for transmission in the RoF-based WLAN is equal to that in the legacy WLAN, $N_{\mathrm{S}}^{\mathrm{R}}=N_{\mathrm{S}}^{\mathrm{L}}$. Thus, the estimated value of $\alpha$ satisfying (15), which should be used for initial value of $\alpha$, is obtained from (16) as

$$
\alpha_{0}=\frac{N_{\mathrm{A}}^{\mathrm{L}}}{N_{\mathrm{A}}^{\mathrm{L}}+N_{\mathrm{S}}^{\mathrm{L}}},
$$

which is a ratio of the number of legacy APs to the total number of nodes in the legacy WLANs. Since CSMA/CA allows each contending node to obtain the same amount of transmission opportunity and the ATRAS scheme is triggered by frames transmitted by legacy nodes, the RoF AP using the ATRAS scheme can obtain $\alpha C\left(N_{\mathrm{A}}^{\mathrm{L}}+\right.$ $N_{\mathrm{S}}^{\mathrm{L}}$ ) transmission opportunity, where $C$ is a transmission opportunity obtained by a node. Thus, when using $\alpha_{0}$ obtained from (17), the RoF AP obtains $C N_{\mathrm{A}}^{\mathrm{L}}$ transmission opportunity, which is equal to the total number of transmission opportunity obtained by legacy APs.

\subsubsection{Adaptive control procedure by $\alpha$}

We now describe the adaptive control procedure and $\alpha$. The term $\alpha_{t+1}$, which represents the value of $\alpha$ at time $t+1(t \geq 1)$, can be derived as follows:

$$
\begin{aligned}
\alpha_{t+1} & =\alpha_{t}-\Delta \alpha_{t}, \\
\Delta \alpha_{t} & =\frac{A}{1+\mathrm{e}^{-g\left(S^{\mathrm{R}}(t)-S^{\mathrm{L}}(t)\right)}}-\frac{A}{2},
\end{aligned}
$$

where $\Delta \alpha_{t}$ refers to a variation of $\alpha$. (19) uses the sigmoid function [31]; $S^{\mathrm{R}}(t)$ and $S^{\mathrm{L}}(t)$ represent the estimated throughput of an RoF and legacy WLAN from $t-1$ to $t$, the units of $S^{\mathrm{R}}(t)$ and $S^{\mathrm{L}}(t)$ are bit/s, $g$ indicates the gain of the sigmoid function, and $A$ is a constant that determines the range of $\Delta \alpha_{t}$, i.e., $-A / 2<$ $\Delta \alpha_{t}<A / 2$. The throughput of both WLANs can be estimated by the RoF AP, which overhears the frame headers and counts the frames transmitted in each WLAN. The RoF AP regularly updates the value of $\alpha$ using (18) and (19) based on the estimated throughput. The details of this procedure are given in Algorithm 1 .

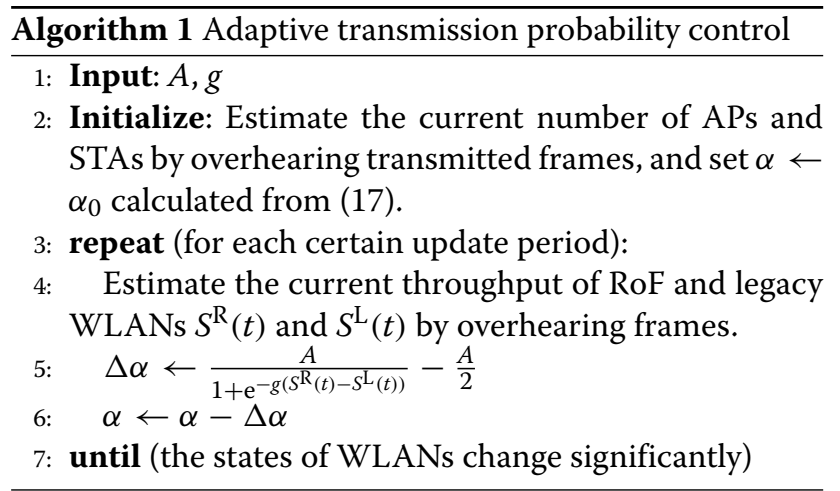


We now explain how to determine the value of $g$. When $\left|S^{\mathrm{R}}(t)-S^{\mathrm{L}}(t)\right|$ is an infinitesimal value, then $\left|\Delta \alpha_{t}\right|$ should be 0 . The wireless throughput may fluctuate for various reasons, and thus we do not want to change $\alpha$ in response to small throughput fluctuations. If we presume that the throughput fluctuations are less than $1 \mathrm{kbit} / \mathrm{s}$, we set $g$ to a value, such as $10^{-5}$ so that $\mathrm{e}^{-g \cdot 10^{3}} \simeq 1$. In contrast, when $\left|S^{\mathrm{R}}(t)-S^{\mathrm{L}}(t)\right|$ is large, $\left|\Delta \alpha_{t}\right|$ should be set to a large value in order to quickly approach the target value.

In this context, $A$ determines the range of $\Delta \alpha_{t}(-A / 2<$ $\left.\Delta \alpha_{t}<A / 2\right)$. The value of $A$ should be selected so as to satisfy $|A|<2$ because $0<\alpha<1$. The optimal value of $A$ depends on $g$, the number of nodes in the RoF and legacy WLAN, and the traffic offered to each node. In this paper, the optimal value of $A$ was determined empirically based on the simulation results.

Since the RoF AP changes the transmission probability based on the measured throughput values, even when the number of users changes, it is possible to control the system such that the throughputs of the RoF WLAN and legacy WLAN are equal. In an asymmetric system configuration, such as an unequal number of users in the RoF WLAN and legacy WLAN, fairness is improved by changing the control target to the ratio of the number of users.

\section{Performance evaluation}

\subsection{Simulation setup}

We evaluated the performance of the ATRAS scheme using the network simulator QualNet [13], which is a reliable simulator used in many literature to evaluate wireless network performance. The simulation model consisted of an RoF-based WLAN and a legacy WLAN, and each had an AP with nine associated STAs, i.e., $N_{\mathrm{A}}^{\mathrm{R}}=N_{\mathrm{A}}^{\mathrm{L}}=1$ and $N_{\mathrm{S}}^{\mathrm{R}}=N_{\mathrm{S}}^{\mathrm{L}}=9$. These WLANs were operated based on the IEEE 802.11a standard and the PHY/MAC parameters shown in Table 1. We also assumed that there were no hidden nodes. We employed the TCP SACK algorithm using a delayed ACK and the Nagle Algorithm, and FTP was chosen as the application protocol. The maximum

Table 1 PHY/MAC parameters

\begin{tabular}{ll}
\hline Parameters & Values \\
\hline SIFS & $16 \mu \mathrm{s}$ \\
DIFS & $34 \mu \mathrm{s}$ \\
Slot time & $9 \mu \mathrm{s}$ \\
Data rate & $54 \mathrm{Mbit} / \mathrm{s}$ \\
MAC payload & 1500 bytes \\
CWmin & 15 \\
CWmax & 1023 \\
Retry limit & 7 \\
\hline
\end{tabular}

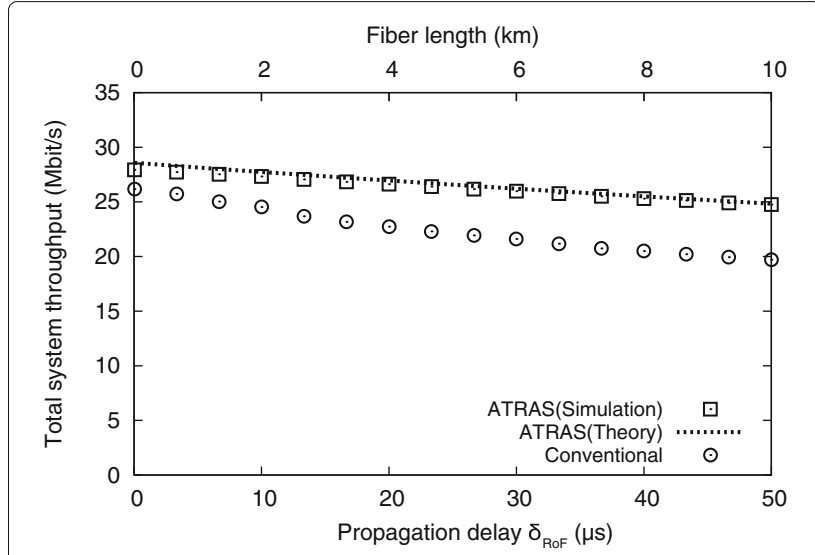

Fig. 7 Total system throughput vs. $\delta_{\text {RoF }}$ with UDP bidirectional full buffer traffic when $\alpha=1$

segment size was 146 bytes, and the sending/receiving buffer size was 64 Kbytes. The ACK/CTS timeout value $T_{\text {timeout }}$ was defined as $T_{\text {timeout }}=T_{\text {st }}+2 \delta_{\text {RoF }}$. The maximum optical fiber length was simulated at $10 \mathrm{~km}$, which corresponded to a $\delta_{\text {RoF }}$ of $50 \mu \mathrm{s}$. The propagation delay between the local nodes $\delta$ was negligibly small, so $\delta$ was set to zero.

In the ATRAS scheme, the RoF STAs and legacy WLAN nodes transmitted frames using the RTS/CTS exchange protocol, and the RoF-based WLAN employed the NAV extension method mentioned in Section 3.3 to solve the ACK frame collision problem in 2.2.2. In the PBA scheme, the RoF AP transmitted the DATA frames using the PBA scheme instead of the ATRAS scheme. In the conventional scheme, the local nodes and RoF AP transmitted frames using the standard CSMA/CA protocol with the RTS/CTS exchange option, and the RoF-based WLAN employed the NAV extension method. In the slot time adjustment scheme, the RoF-based WLAN used an extended slot time and the legacy WLAN used the standard slot time defined

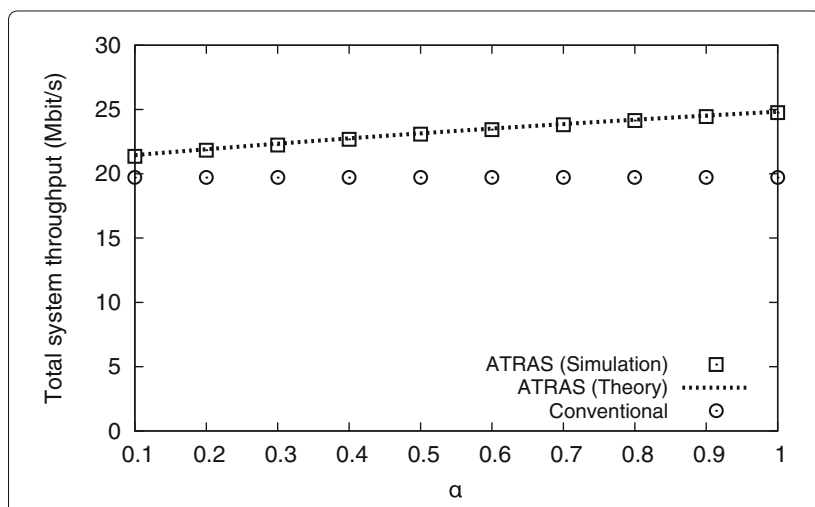

Fig. 8 Total system throughput vs. $\alpha$ with UDP bidirectional full buffer traffic when $\delta_{\text {RoF }}=50 \mu \mathrm{s}$ 


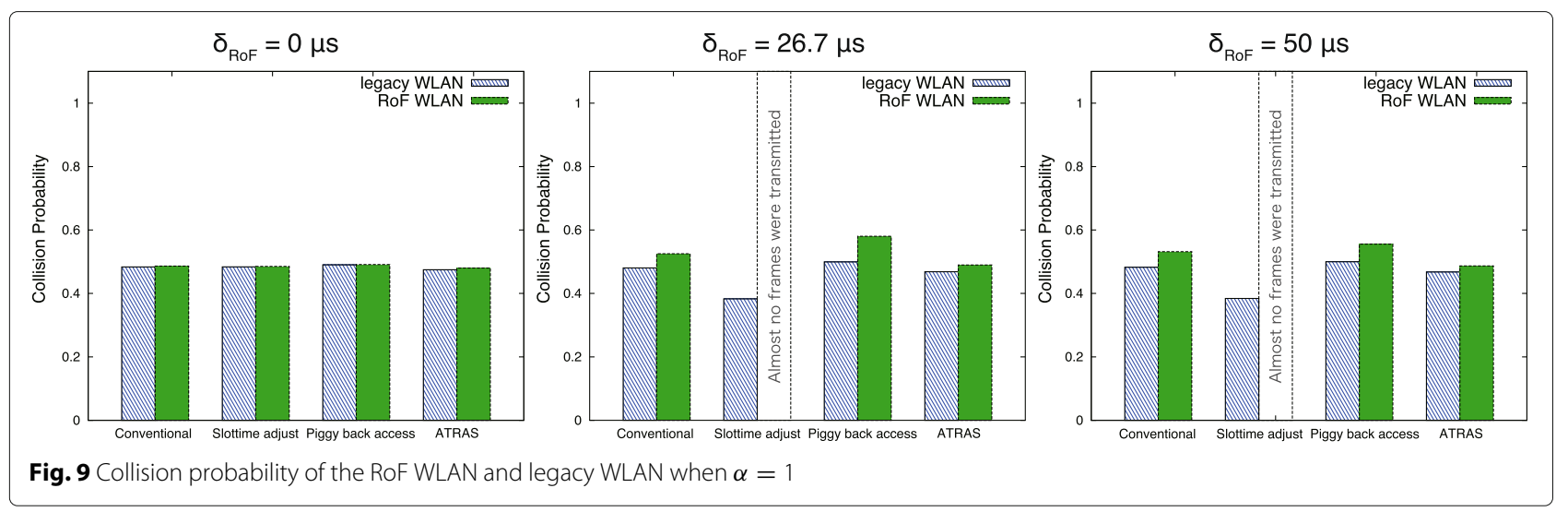

in the IEEE 802.11a standard. The extended slot time was $2 \delta_{\mathrm{RoF}}+4 \mu \mathrm{s}$.

\subsection{Simulation and numerical results}

We evaluated the performance of the MAC and transport layers. In the MAC layer performance evaluation, we considered UDP bidirectional full buffer traffic and confirmed that the theoretical throughput performance was in good agreement with the simulation results. We also compared the frame collision probability in the MAC layer performance evaluation. When evaluating the performance of the transport layer, we compared the performance of the ATRAS scheme with that of the conventional CSMA/CA scheme, slot time adjustment scheme, and the PBA scheme with TCP downlink traffic.

\subsubsection{MAC-layer performance}

Figure 7 shows the total system throughput of the RoFbased WLAN and legacy WLAN as a function of the propagation delay $\delta_{\mathrm{RoF}}$ with $\alpha=1$. When $\alpha=1$, the RoF AP transmits a frame every time the RoF AP overhears a CTS frame transmitted by a legacy AP or a legacy STA. The ATRAS scheme achieved a higher throughput than the conventional scheme. In the both schemes, the total throughput decreases as the propagation delay $\delta_{\text {RoF }}$ increases since the required time to exchange frames in RoF-based WLAN increases. In addition to the throughput degradation, increase in frame collision degrades throughput in the conventional scheme, which is the reason why the throughput gain of the proposed scheme increases as $\delta_{\text {RoF }}$ increases.

Figure 8 shows the total system throughput as a function of transmission probability $\alpha$, where $\delta_{\text {RoF }}=50 \mu \mathrm{s}$. The ATRAS scheme can control the throughput of the RoF-based WLAN by adjusting $\alpha$. Moreover, in both of the figures, the simulation results of the ATRAS scheme are in agreement with the theoretical values.

Figure 9 shows the collision probability of frames as a function of the propagation delay $\delta_{\mathrm{RoF}}$ when $\alpha=1$ in the ATRAS scheme. Since the RoF WLAN with slot time adjustment scheme cannot transmit frames when $\delta_{\text {RoF }}$ is longer than $26.7 \mu$ s due to long DIFS and backoff, the collision probability of the RoF WLAN with slot time adjustment is not shown in the figure. When the ATRAS scheme is used, the collision probability of the RoF WLAN does not increase as $\delta_{\text {RoF }}$ increases, while the probabilities in the other schemes increase. The average collision probability of both WLANs also increases in the scheme

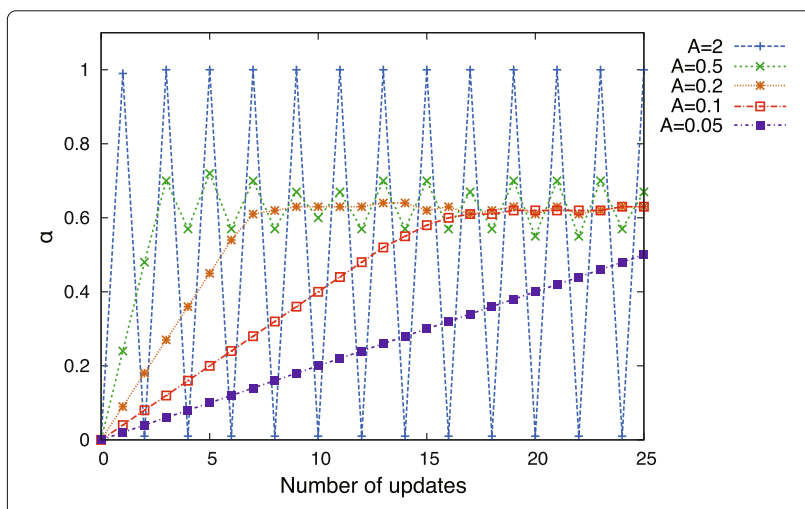

Fig. 10 Time transition of $\alpha$ parameterized by $A$ when $\delta_{\text {RoF }}=50 \mu \mathrm{s}$

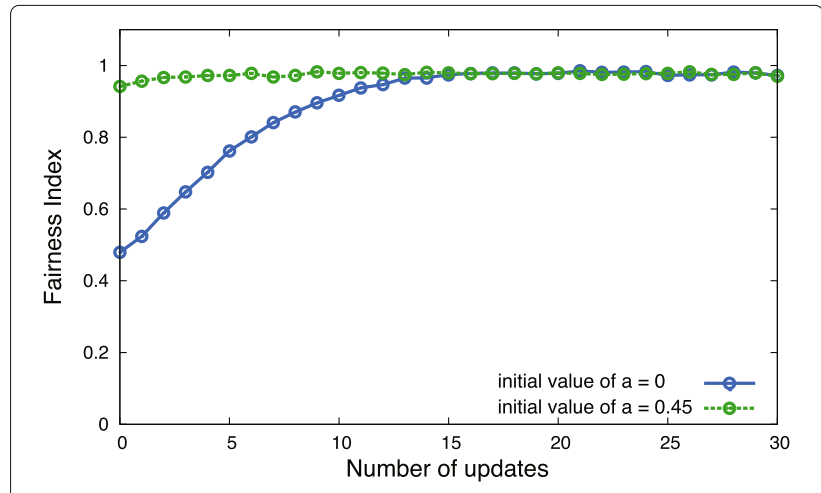

Fig. 11 Fairness index of TCP downlink flow 

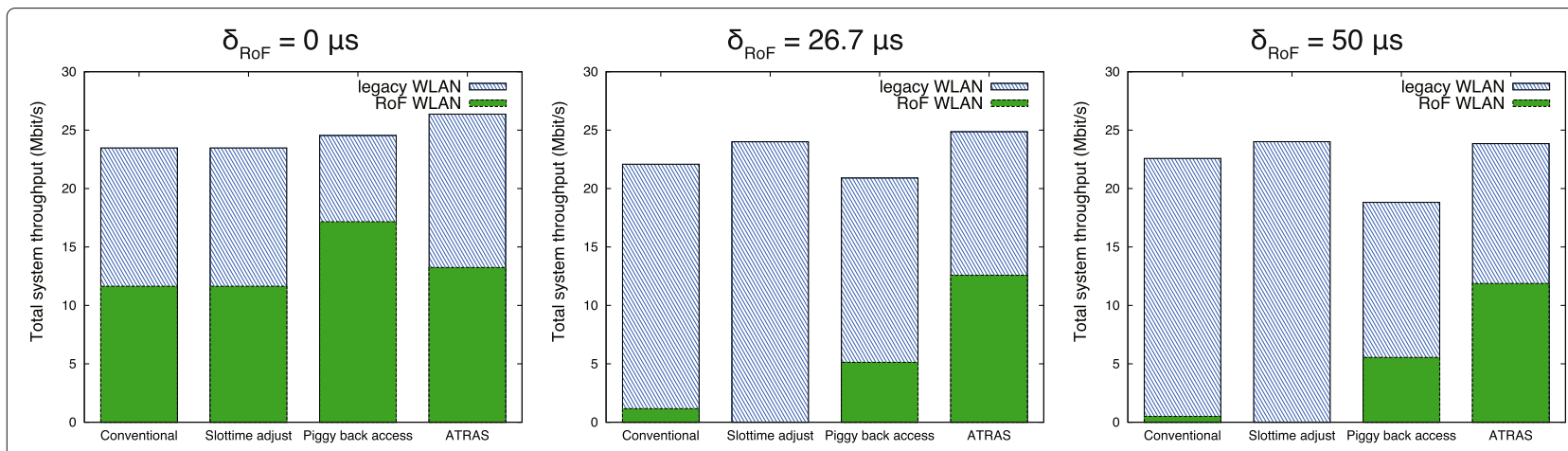

Fig. 12 Total throughput of the RoF-based WLAN and legacy WLAN

other than the ATRAS scheme as $\delta_{\text {RoF }}$ increases. This is because the frame collision due to delay mentioned in Section 2.2.1 occurs frequently when $\delta_{\text {RoF }}$ is long. In the ATRAS scheme, the RoF AP can transmit frames without collision, which enables WLANs to maintain lower collision probability when $\delta_{\mathrm{RoF}}$ is long.

\subsubsection{Transport layer performance}

In this scenario, we considered the TCP downlink traffic in both the RoF and legacy WLANs. First, we evaluated the convergence of $\alpha$ with the adaptive control scheme. We determined the gain of the sigmoid function $g$ and the constant value $A$ in (19). The update of $\alpha$ was performed every time the RoF AP received 100,000 TCP-DATA packets transmitted by the legacy AP and legacy STAs. As we discussed in 4.3.3, $g$ was set to $g=10^{-6}$ in the simulation.

Figure 10 shows the time dependence of $\alpha$ parameterized by $A$. The horizontal axis indicates a number of updates to $\alpha$. In the cases where $A=2$ and $A=0.5$, the value of $\alpha$ fluctuated owing to the large value of $A$. On the other hand, if we set the value of $A$ to be small, such as $A=0.05$, then $\alpha$ converged slowly. Based on these simulation results, we set $A=0.1$.

Next, we evaluated the convergence time of $\alpha$ with the adaptive control scheme in order to clarify the effectiveness of our initial value determination. As was mentioned above, we set $g=10^{-6}$ and $A=0.1$ in (19). From (17), $\alpha_{0}$ was set to 0.45 in this scenario. We compared the proposed scheme to a scheme where $\alpha_{0}=0$.

Figure 11 shows the Jain's fairness index [32], which is defined as

$$
F=\frac{\left(\sum_{i=1}^{n} \theta_{i}\right)^{2}}{n \sum_{i=1}^{n} \theta_{i}^{2}},
$$

where $n$ is the total number of downlink flows in the RoF and legacy WLANs, and $\theta_{i}$ is the TCP throughput of the $i$ th connection. As shown in Fig. 11, the fairness index is close to one as a result of using the proposed adaptive control procedure. Moreover, it takes less time to achieve fairness by using the initial value $\alpha_{0}=0.45$ derived from (17) as compared to the time required when $\alpha_{0}=0$.

Next, we compared the throughput of the ATRAS scheme with that of the conventional CSMA/CA scheme, the slot time adjustment scheme, and the PBA scheme. Figures 12 and 13 show the system throughput of each WLAN and the fairness index based on the value of $\delta_{\text {RoF }}$. The ATRAS scheme was able to increase the throughput of the RoF-based WLAN and achieve fairness regardless of the $\delta_{\text {RoF }}$ value. This is because the ATRAS scheme gives collision-free transmission opportunity to the RoF AP, and the adaptive transmission probability control adjusts $\alpha$ to increase the throughput of the RoF AP up to the same level as the legacy WLAN. Moreover, the total system throughput in the proposed scheme was higher than in the other schemes because the number of frame collisions caused by the propagation delay was reduced. Using the CSMA/CA scheme, the throughput of the RoF-based WLAN decreased and the fairness index also decreased as the $\delta_{\text {RoF }}$ increased. When the RoF-based WLAN used the slot time adjustment scheme, the throughput of the RoF-based WLAN approached zero if $\delta_{\mathrm{RoF}} \geq 10 \mu \mathrm{s}$. This is because the nodes in the RoF-based WLAN have much

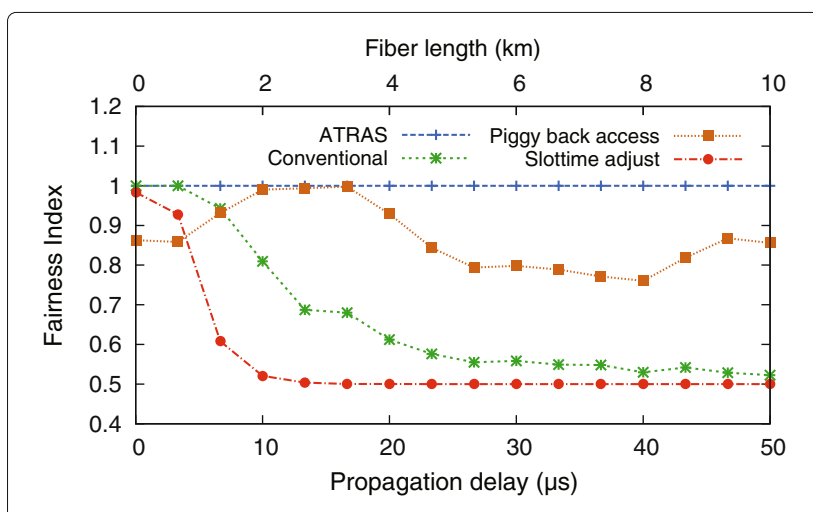

Fig. 13 Fairness Index when the RoF AP used each scheme 
longer backoff periods than those in the legacy WLAN, which means that they cannot transmit enough frames for the reasons described in Section 3.1. The use of the PBA scheme was able to improve the throughput of the RoF-based WLAN compared to the throughput in the conventional CSMA/CA scheme, because the RoF AP was able to transmit its frames using both the CSMA/CA and PBA schemes. The throughput of the RoF-based WLAN, however, decreased based on $\delta_{\text {RoF }}$ as a consequence of the increase in frame collisions.

\section{Conclusions}

In this paper, we proposed the ATRAS scheme to reduce unexpected frame collisions and improve fairness between an RoF-based WLAN and legacy WLANs in a coexisting environment. The ATRAS scheme enables the RoF AP to transmit its frames in advance so that the frames do not collide with other frames, and the adaptive transmission probability control is able to achieve fairness between the RoF-based WLAN and legacy WLANs. Our simulation and numerical results demonstrated the performance of the proposed scheme, which are highlighted as follows: The proposed scheme increases MAC layer throughput by $12.7-24.6 \%$ and decreases frame collision probability compared to the conventional scheme. The proposed adaptive transmission probability control converges quickly within a few updates. The proposed scheme increases the TCP throughput of the RoF-based WLAN up to the same level as that in the legacy WLAN and achieves fairness regardless of propagation delay.

Our future work includes applying the proposed protocol to environments where IEEE $802.11 \mathrm{n} / \mathrm{ac}$ is used for WLANs or where multiple RoF-based WLANs and legacy WLANs coexist and extending the proposed protocol to use multiple medium access schemes adaptively as mentioned in Section 4.1.

\section{Competing interests}

The authors declare that they have no competing interests.

\section{Publisher's Note}

Springer Nature remains neutral with regard to jurisdictional claims in published maps and institutional affiliations.

\section{Author details}

${ }^{1}$ Graduate School of Informatics, Kyoto University, Kyoto, Japan. ${ }^{2}$ NTT Network Innovation Laboratories, NTT Corporation, Yokohama, Japan.

Received: 30 October 2016 Accepted: 27 June 2017

Published online: 19 July 2017

\section{References}

1. SK Yong, C-C Chong, An overview of multigigabit wireless through millimeter wave technology: potentials and technical challenges. EURASIP J. Wirel. Commun. Netw. 2007(1), 1-10 (2006)

2. JA Gutierrez, M Naeve, E Callaway, M Bourgeois, V Mitter, B Heile, IEEE 802.15. 4: a developing standard for low-power low-cost wireless personal area networks. IEEE Netw. 15(5), 12-19 (2001)
3. E Khorov, A Lyakhov, A Krotov, A Guschin, A survey on IEEE 802.11 ah: an enabling networking technology for smart cities. Comput. Commun. 58, 53-69 (2015)

4. R Riggio, A Bradai, D Harutyunyan, T Rasheed, T Ahmed, Scheduling wireless virtual networks functions. IEEE Trans. Netw. Serv. Manag. 13(2), 240-252 (2016)

5. H Al-Raweshidy, S Komaki, Radio over fiber technologies for mobile communications networks. (Artech House, London, 2002)

6. L Maksymiuk, M Kowalczyk, J Siuzdak, Multimode fiber passive optical network for IEEE 802.11 signal distribution. J. Opt. Commun. Netw. 6(8), 743-753 (2014)

7. D Novak, RB Waterhouse, A Nirmalathas, C Lim, PA Gamage, TR Clark, ML Dennis, JA Nanzer, Radio-over-fiber technologies for emerging wireless systems. IEEE J. Quantum Electron. 52(1), 1-11 (2016)

8. M Zhu, L Zhang, J Wang, L Cheng, C Liu, G-K Chang, Radio-over-fiber access architecture for integrated broadband wireless services. J. Light. Technol. 31(23), 3614-3620 (2013)

9. J Beas, G Castanón, I Aldaya, A Aragón-Zavala, G Campuzano, Millimeter-wave frequency radio over fiber systems: a survey. IEEE Commun. Surv. Tutor. 15(4), 1593-1619 (2013)

10. S Deronne, V Moeyaert, S Bette, in Proc. IEEE SCVT. Analysis of the MAC performances in $802.11 \mathrm{~g}$ radio-over-fiber systems, (Ghent, Belgium, 2011), pp. 1-5

11. S Deronne, V Moeyaert, $S$ Bette, in Proc. ONDM. WiFi transmission in radio-over-fiber systems: performance of the IEEE $802.11 \mathrm{n}$ aggregation mechanism, (Brest, France, 2013), pp. 167-172

12. S Zhang, D Franklin, in Proc. IEEE ICCC. Feasibility study on the implementation of IEEE 802.11 on cloud-based radio over fibre architecture, (Sydney, Australia, 2014), pp. 2891-2896

13. QualNet. http://web.scalable-networks.com/qualnet-networksimulator-software. Accessed 7 July 2017

14. IEEE Std. IEEE Standard for Information technology-Telecommunications and information exchange between systems Local and metropolitan area networks-Specific requirements - Part 11: Wireless LAN Medium Access Control (MAC) and Physical Layer (PHY) Specifications, in IEEE Std 802.11-2016, pp.1-3534, doi:10.1109/IEEESTD.2016.7786995. http:// ieexplore.ieee.org/document/7786995/

15. GP Agrawal, Nonlinear fiber optics. (Academic press, Massachusetts, 2007)

16. T Nishio, K Funabiki, M Morikura, K Yamamoto, D Murayama, K Nakahira, MAC protocol for improving throughput and balancing uplink/downlink throughput for wireless local area networks with long propagation delays. IEICE Trans. Commun. E100.B(5), 874-883 (2017)

17. Z Zeng, Y Gao, K Tan, PR Kumar, in Proc. IEEE INFOCOM. CHAIN: introducing minimum controlled coordination into random access MAC, (Shanghai, China, 2011), pp. 2669-2677

18. F Wamser, R Pries, D Staehle, K Heck, P Tran-Gia, Traffic characterization of a residential wireless internet access. Telecommun. Syst. 48(1-2), 5-17 (2011)

19. Q Wei, I Aad, L Scalia, J Widmer, P Hofmann, L Loyola, E-MAC: an elastic MAC layer for IEEE 802.11 networks. Wirel. Commun. Mob. Comput. 13(4), 393-409 (2013)

20. Y Khan, M Derakhshani, S Parsaeefard, T Le-Ngoc, in Proc. IEEE Globecom Workshops. Self-organizing TDMA MAC protocol for effective capacity improvement in IEEE 802.11 WLANs, (California, 2015), pp. 1-6

21. G Jakllari, M Neufeld, R Ramanathan, in Proc. IEEE MASS. A framework for frameless tdma using slot chains, (Nevada, 2012), pp. 56-64

22. P Djukic, P Mohapatra, Soft-TDMAC: a software-based 802.11 overlay TDMA MAC with microsecond synchronization. IEEE Trans. Mob. Comput. 11(3), 478-491 (2012)

23. M Tuysuz, HA Mantar, A beacon-based collision-free channel access scheme for IEEE 802.11 WLANs. Wirel. Pers. Commun. 75(1), 155-177 (2014)

24. MF Tuysuz, HA Mantar, Exploiting the channel using uninterrupted collision-free MAC adaptation over IEEE 802.11 WLANs. Wirel. Commun. Mob. Comput. 15(5), 889-909 (2015)

25. J Barcelo, B Bellalta, C Cano, M Oliver, in Proc. Eunice Open European Summer School. Learning-BEB: avoiding collisions in wlans, (Brest, France, 2008), pp. 1-8

26. NetComm Wireless HS1200N. http://www.netcommwireless.com/sites/ default/files/HS1200N-User-Manual.pdf. Accessed 7 July 2017 
27. ath9k driver. https://wireless.wiki.kernel.org/en/users/drivers/ath9k. Accessed 7 July 2017

28. M Derakhshani, T Le-Ngoc, in Proc. IEEE Globecom. Adaptive access control of CSMA/CA in wireless lans for throughput improvement, (Georgia, USA, 2013), pp. 2951-2955

29. G Bianchi, Performance analysis of the IEEE 802.11 distributed coordination function. IEEE J. Sel. Areas Commun. 18(3), 535-547 (2000)

30. R Bruno, M Conti, E Gregori, in Proc. ACM MSWiM. Performance modelling and measurements of TCP transfer throughput in 802.11-based WLAN (Malaga, Spain, 2006), pp. 4-11

31. WA Little, The existence of persistent states in the brain. (Science \& Business Media, US, 1974), pp. 145-164

32. R Jain, D-M Chiu, WR Hawe, A quantitative measure of fairness and discrimination for resource allocation in shared computer system. DEC Research Report TR-301 (1984). https://jwcn-eurasipjournals. springeropen.com/articles/10.1186/s13638-015-0340-3

\section{Submit your manuscript to a SpringerOpen ${ }^{\circ}$ journal and benefit from:}

- Convenient online submission

- Rigorous peer review

- Open access: articles freely available online

- High visibility within the field

- Retaining the copyright to your article

Submit your next manuscript at $\boldsymbol{s p r i n g e r o p e n . c o m ~}$ 